\title{
From Centralized Systems to Distributed 5G-enabled Manufacturing
}

\author{
Kathryn Baxter ${ }^{\mathrm{a}, *}$, John Lundberg ${ }^{\mathrm{b}}$ \\ ${ }^{a}$ Department of Automotive Technology, Rio Hondo College, Whittier, California, USA \\ ${ }^{b}$ Department of computer engineering, Mount San Antonio College, Walnut, CA. USA
}

\begin{abstract}
The landscape of centralized cloud computing is now changing to distributed and decentralized clouds with promising impacts on energy consumption, resource availability, resilience, and customer experience. This research highlights the impacts of emerging IT trends, namely, 5G wireless technology, blockchain, and industrial Artificial Intelligence (AI) in development and realization of the next generation of cloud computing. Integration of these technologies in cyber-physical system and cloud manufacturing paradigms is explained and a unified edge-fog-cloud architecture is proposed for successful implementation in manufacturing systems.
\end{abstract}

Keywords: 5G Wireless Technology; Industrial Artificial Intelligence; Blockchain; Cloud Computing; Cyber-Physical System; Cloud Manufacturing.

\section{Introduction}

Centralized cloud infrastructure is used in the most of industrial application for providing services to clients. Although using a centralized cloud and a single provider has obvious advantages, there are some important challenges that need to be addressed. For example, centralized clouds may consume between $14 \%$ and $25 \%$ more energy than distributed clouds [1]. They are susceptible to a single point of failure [2] and lack scalability. Moreover, cloud centers may be geographically distant from the data source, thereby posing limitations on data transmission performance which could be significantly important for sensitive applications. Realization of cloud computing in manufacturing, Cloud Manufacturing (CM), leads to an interconnected service-oriented network where manufacturing resources are encapsulated and offered as services to different clients. Advances in Cyber-Physical Systems (CPS) facilitates interconnectivity, resource availability, and manufacturing productivity. In smart manufacturing paradigm, both CPS and CM are integrated to provide seamless services to clients both inside and outside of the factory and realize a unified customer and product-centric platform. Successful implementation of CPS and CM requires advanced technologies for a) reliable and efficient data collection, manipulation, and transfer, b) Dynamic collaboration, resource sharing, and management, c) Autonomous decision making, d) Transparent provision of information, and e) Secure and reliable peer to peer (P2P) interactions. In order to address these challenges, authors in [3] highlighted the contribution of industrial Artificial Intelligence (AI) in enhancing transparency and decision making, and authors in [4] proposed fog computing as an enabler for on-demand resource access and efficient networking. Authors in [5] proposed the integration of blockchain with 5C-CPS architecture to facilitate P2P interactions and secure interconnectivity. Using $5 \mathrm{G}$ wireless technology in the realization of cloud manufacturing has been discussed in [6,7]. Although these studies address some of the challenges in the realization of smart manufacturing, still there is a need for a systematic framework and model to integrate these emerging technologies in CPS and CM paradigms and clarify their significant contributions and functionalities.

A summary of the core technology and key advantages of 5G, blockchain, industrial AI, and distributed clouds in the realization of smart manufacturing is presented in Fig. 1 and will be explained in the rest of the paper.

\footnotetext{
${ }^{*}$ Corresponding author

Email address: kathryn.baxter0274@my.riohondo.edu (Kathryn Baxter)
} 


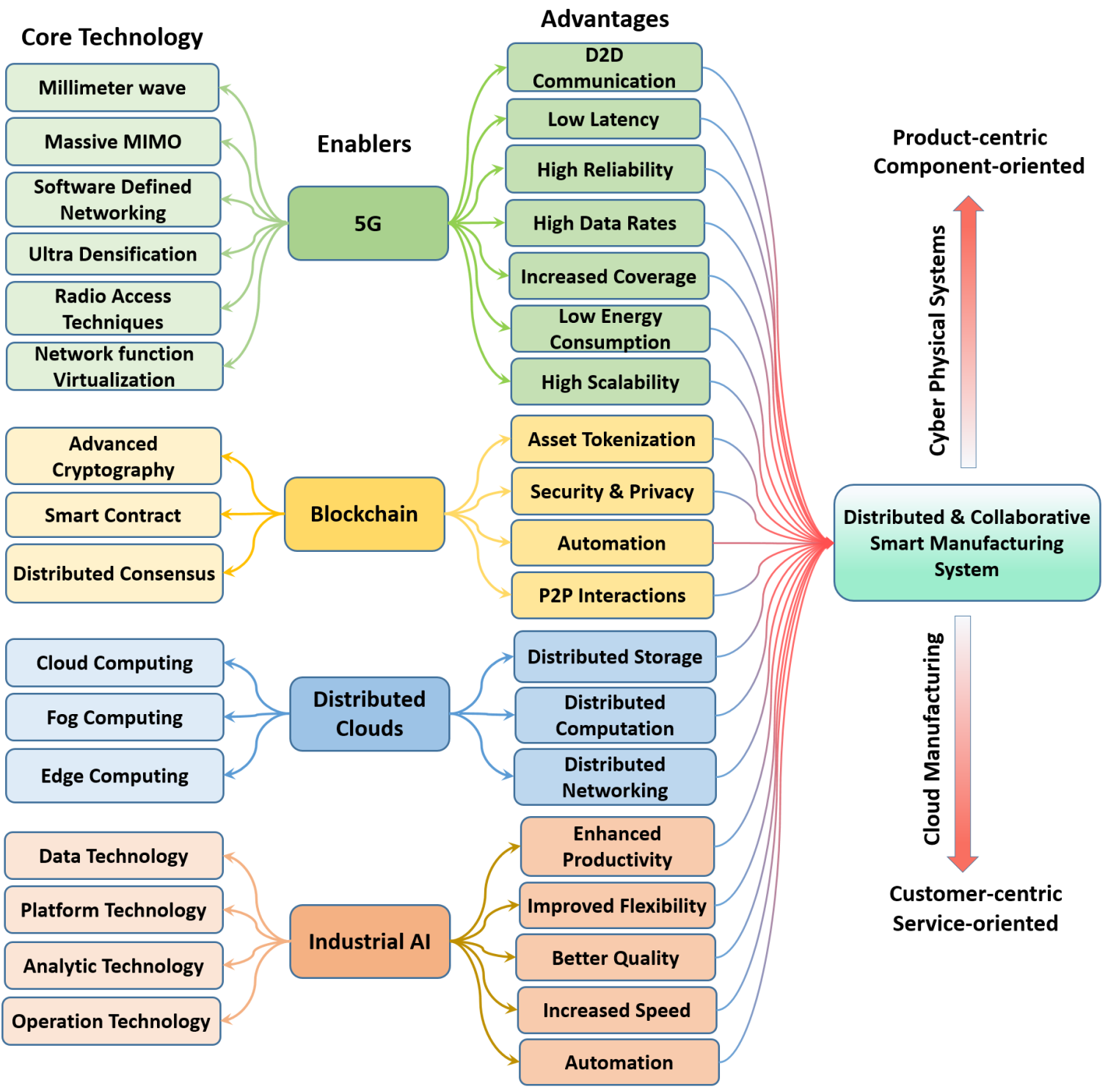

Figure 1: Emerging IT trends and their contributions in smart manufacturing. 


\section{5G enabled cyber-physical systems}

Any attempt to generate a cyber-physical integrated system requires real-time data collection from different assets and ubiquitous connectivity that facilitates real-time data flow with high reliability, minimum latency, high coverage, and high data rate. Although telecommunication technologies have been examined as a potential solution in the last decades, due to their low performance, they have not played a major role in industrial applications. However, the fifth wireless technology (i.g. 5G) with inherent advantages is seen as a real game-changer. As depicted in Fig. 1, the core enabling technologies of $5 \mathrm{G}$ can be summarized in 1) Millimeter-wave [8], 2) Massive MIMO [9], 3) Software Defined Networking [10], 4) Ultra Densification [11] 5) Radio Access Network [12] and 6) Network function Virtualization [13]. A comprehensive study on the roles and functionalities of these technologies in development of $5 \mathrm{G}$ is given in [14,15]. Depending on the application, the use cases of $5 \mathrm{G}$ can be summarized in three main categories [16], namely mMTC (massive Machine Type Communications), eMBB (enhanced Mobile Broadband), and URLLC (Ultra-Reliable and Low Latency Communication), as discussed below:

\section{1. $m M T C$}

This scenario focuses on providing wireless connectivity to a massive number of devices that often are sporadically active, have limited power capacity, and send small data payloads [17]. This implementation improves interconnectivity, scalability, coverage, and indoor penetration and therefore may provide ubiquitous connectivity for IoT devices and facilitate data collection across all devices in the manufacturing shop floor.

\section{2. $U R L L C$}

In this scenario, the accent is on latency and reliability in data transmission. The objective is to transfer a small payload of data with ultra-low latency and high reliability [18]. Possible applications of URLLC in manufacturing are autonomous shop floor, remote control, and mission-critical applications.

\section{3. $e M B B$}

In this scenario, the data rate is maximized with moderate reliability to transfer large payloads of data. Applications that require high data rates such as augmented reality, intensive collaborative work such as 3D design, and digital twin would significantly benefit from this implementation.

\section{Blockchain}

Blockchain, a distributed ledger, is a decentralized network of multiple participants who achieve consensus over a dataset. The ledger is constantly updated and shared between the members of a peer to peer (P2P) network [19]. Blockchain can provide trustworthy and tamper-resistant transparent information about different stages of the product life cycle [20] which helps in tracing the materials and improving resource allocation. Blockchain enhances security and privacy of distributed networks [21] and by using smart contracts, different tasks can be executed in an autonomous and decentralized way. Blockchain also facilitates distributed storage [22], asset tokenization [23], micro-payment and resource sharing functions [22,24].

\section{Industrial AI}

Industrial AI is a "systematic discipline for utilization of artificial intelligence in industrial applications for knowledge discovery and problem-solving with a sustainable performance". Implementation of industrial AI in a distributed and decentralized network increase manufacturing resilience, fault tolerance, and efficiency. A network of AI agents brings self-awareness, self-predict, self-adapt, and self-configure functionalities to the elements of the system. Blockchain tools provide mutual authentication between devices, evaluate the legitimacy of software and data, and enhance the privacy and security of AI modules. Due to the integration of AI agents with $5 \mathrm{G}$ and blockchain, availability of data increases and more independent AI developers can cooperate in a systematic fashion.

Time-consuming and advanced applications such as deep learning and transfer learning can be realized through the utilization of distributed computing and federated learning. Finally, manufacturing tasks and 


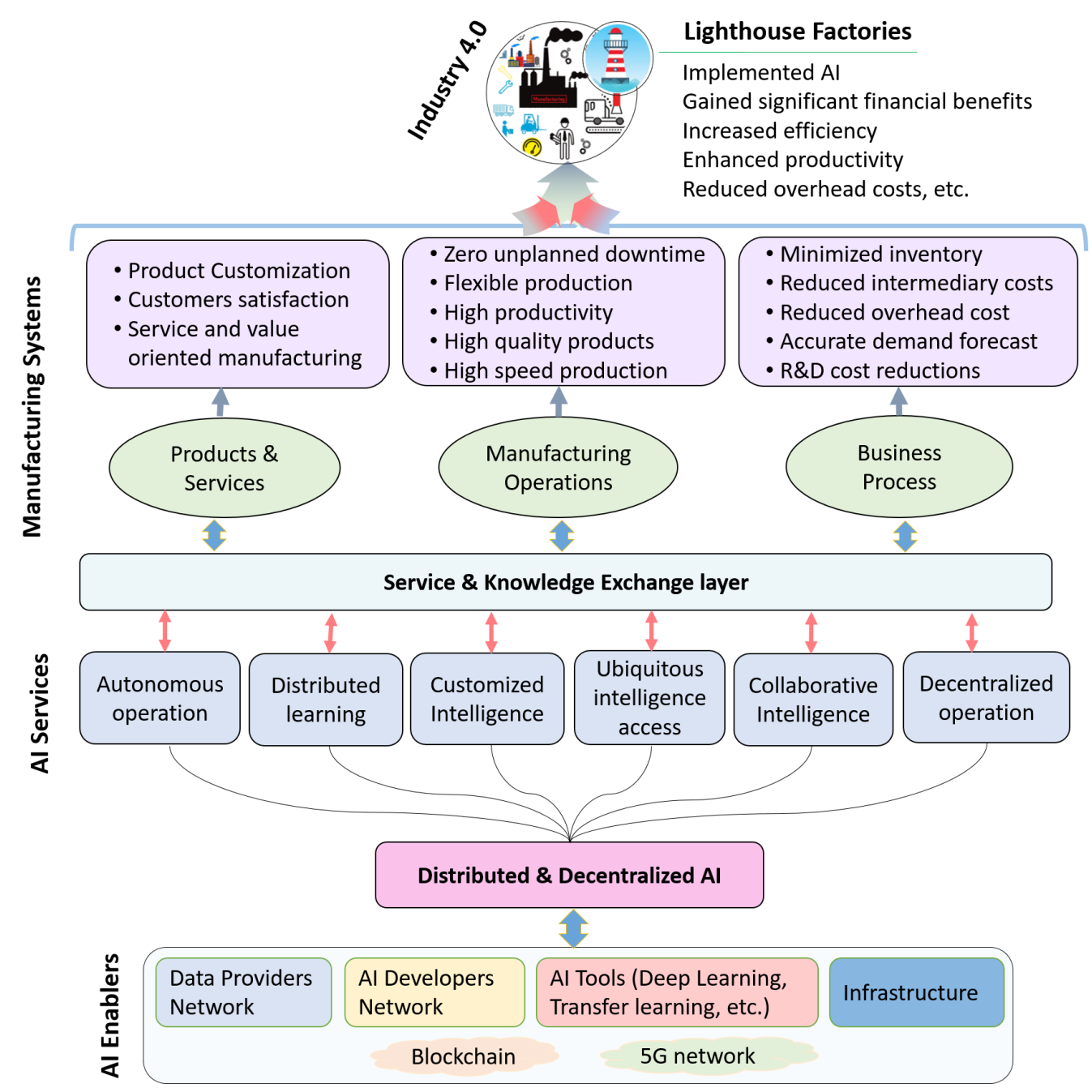

Figure 2: The proposed framework for autonomous smart manufacturing. 
decision making could be achieved in an autonomous and decentralized approach. A conceptual framework showing the proposed agent-based autonomous manufacturing is given in Fig. 3. In this structure, a network of AI enablers is designed to create high-quality AI tools, evaluate their performance and suitability before passing them to the service and knowledge exchange layer for utilization in different applications. In order to create an autonomous intelligent process, advanced AI tools such as deep learning are utilized to extract actionable information from raw data automatically and provide insights for decision making. $5 \mathrm{G}$ advanced connectivity and the blockchain's smart contracts would be of significant use in the automation process.

\section{The proposed edge-fog-cloud architecture in 5G environment}

The next generation of edge-fog-cloud architecture is shown in Fig. 4 where not only resources are brought closer to clients, but also blockchain, 5G, and industrial AI technologies are incorporated in different elements of the network to enhance P2P interactions and realize autonomous manufacturing. 5G wireless technology can be utilized to reduce communication latency, maximizes throughput, increases interconnectivity, and potentially speeds up the computational tasks by enabling distributed computing functionalities. Security and privacy are the main challenges in the realization of distributed clouds which could be addressed through the adaptation of blockchain technology $[21,25,26]$ and finally, industrial AI facilitates autonomous and distributed decision making. Moreover, in the proposed architecture, multi-cloud providers work together and may share their infrastructure in a P2P interconnected network to enhance resource availability and reduce operational expenses. Different elements of the proposed edge-fog-cloud architecture are explained below.

\subsection{Edge Micro-Clouds}

A micro-cloud is a standalone and on-demand resource that has computational, storage, and networking capabilities and is equipped with AI agents for independent and cooperative decision making. Incorporating $5 \mathrm{G}$ in micro-clouds facilitates device to device (D2D) communication without interaction with a base station (BS) or with limited BS involvement. This function creates a massive ad hoc mesh network that significantly increases interconnectivity and brings enormous advantages that didn't exist in manufacturing before. Such network supports mobile applications, easy deployment, high scalability, low latency, and geopolitical independence at a lower cost and restrictions than traditional cloud computing structures. Micro clouds could be essential for delay-sensitive manufacturing applications like real-time monitoring systems [26,27], smart assembly platforms [27], and human-machine integrated data representation [28]. A machine-level Micro-Cloud manages six major aspects (6M) namely, 1- Material: property, strengths, and functions 2- Machine: precision, calibration, and automation 3-Methods: tools, analytics, and knowledge, 4- Measurement: calibration, noise reduction, accuracy, 5- Maintenance: monitor, predict, and avoid 6- Models: predict, optimize, and resilient. All these functionalities could be offered as micro-services to clients at machine-level (e.g. controllers, sensors, etc.), shop floor level (e.g. operators for job and maintenance scheduling), or even at the enterprise level to the machine and component manufacturers. A network of Micro-Clouds can create a cooperative framework for global decision making, peer to peer interactions, and knowledge and resource sharing.

\subsection{Fogs Network}

Although Micro-Clouds network handles low computational tasks and provides low latency services at the edge of the network, there is a crucial need for providing services with higher intelligence requirements and medium access delay without sending data to the cloud servers. Fog layer is designed to address these requirements and manage interactions inside and between different Micro-Clouds. In addition, Fogs network brings context awareness, reduces inter-application isolation, supports real-time streaming, improves the quality of services, and increases resource availability. Using blockchain-based distributed Software Defined Networking will address security and privacy concerns and help in resource allocation and management. In this distributed network, adaptation of $5 \mathrm{G}$ would enhance communication speed, data rate, reliability, and coverage. For integrating $5 \mathrm{G}$ in cloud computing technology, a Heterogeneous Cloud Radio Access Network (H-RAN) was proposed in [29]. Where, Macro Base Stations (MBSs) are used mainly for transferring the control signals and enhancing coverage, and Remote Radio Heads (RRHs) are utilized to provide highspeed data transfer in the user plane. However, RRH in a Fog-based computing network must be evolved 


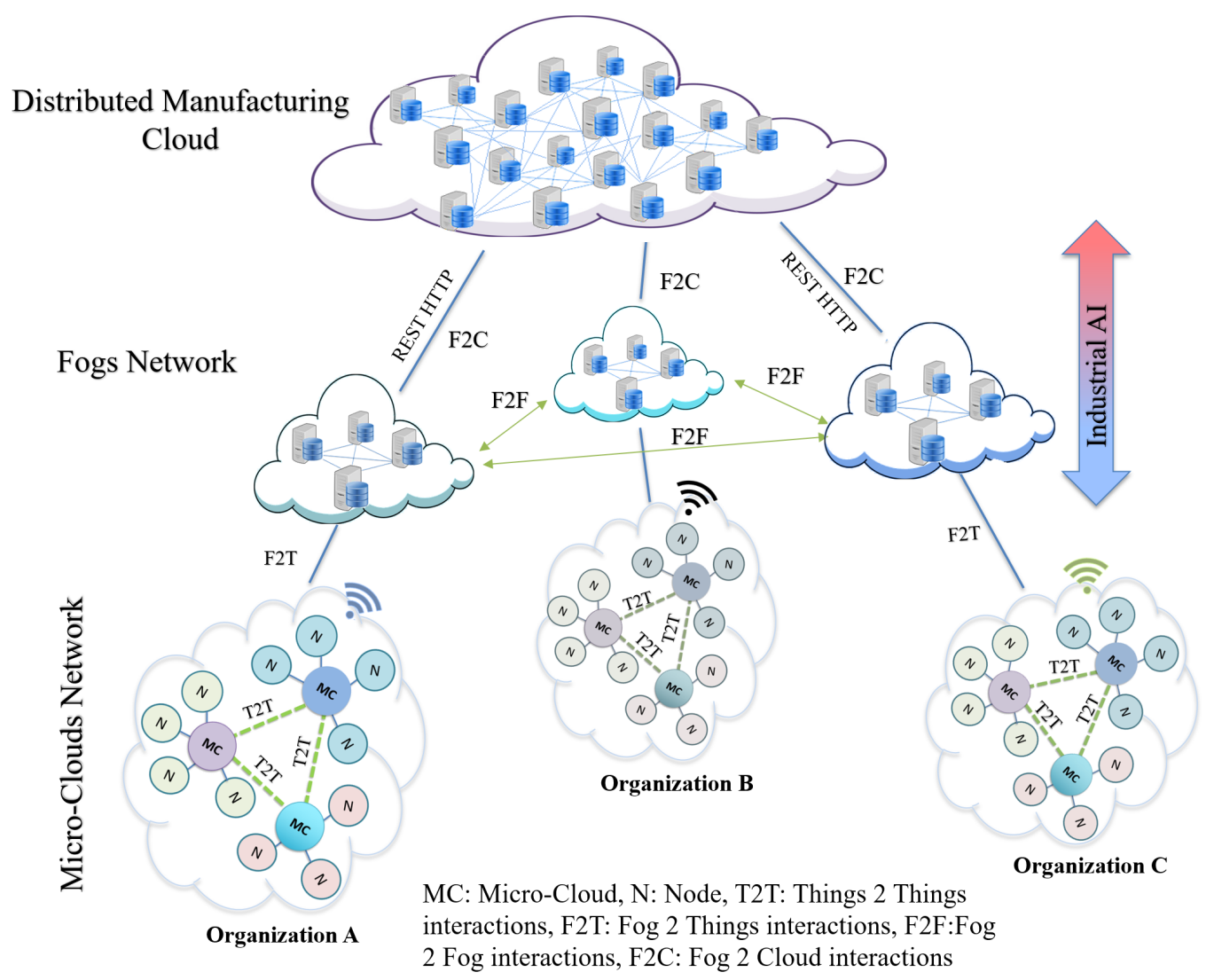

Figure 3: The proposed edge-fog-cloud architecture.

to support collaborative radio signal processing and resource management. Due to the Network Function Virtualization, micro-services can be defined and distributed across multiple resources which results in more efficient resource utilization and networking capacity enhancement.

\subsection{Distributed Manufacturing Cloud}

While Micro-Clouds and Fogs network provide localization and dynamic services for low and medium delay-sensitive applications, a more global service and functionality management network is required for big data storage, advanced analytics, control of cloud-fog interactions, distribution of resources, and provision of large-scale services. A cloud layer also defines all or some of the functionalities required for the Fogs network and monitors them constantly to guarantee a high-quality service provision. A distributed cloud network is spread geographically close to the clients therefore, it has less operational cost and at the same time provides services with lower latency and high bandwidth. Due to distributed data storage and computation across multiple clouds, this design is more fault-tolerant and provides on-demand and resilient resources for a variety of applications. In the proposed blockchain-enabled distributed cloud structure, all providers connect in a P2P network which creates a more secure and competitive network benefiting both cloud providers and clients. Wherein, each client can select different cloud providers from a pool of certified candidates for hosting their data/knowledge, applications, services, etc. By incorporation 5G in this design, a flexible service automation framework is achieved for networking, end-to-end service, and resource orchestration. 5G envisions new services that didn't exist in the previous network generations, such as augmented reality and digital twin. And due to the user-centric functions of $5 \mathrm{G}$, service access and customer experience improve significantly and devices, machines, operators, and products become innovative smart elements that generate and use applications, data, and services. 


\section{Conclusions}

This paper proposes a systematic approach for the integration of $5 \mathrm{G}$ wireless technology, blockchain, and industrial AI in the next generation cloud computing architecture. The key contributions and functionalities of these technologies in the realization of smart manufacturing are discussed. A unified edge-fog-cloud architecture is proposed for providing ubiquitous connectivity, computation, and service access across manufacturing ecosystem.

\section{References}

[1] Ahvar E, Orgerie A-C, Lebre A. Estimating Energy Consumption of Cloud, Fog and Edge Computing Infrastructures. IEEE Trans Sustain Comput 2019:1-1.

[2] Alhamazani K, Ranjan R, Mitra K, Rabhi F, Jayaraman PP, Khan SU, et al. An overview of the commercial cloud monitoring tools: research dimensions, design issues, and state-of-the-art. Computing 2015;97:357-77.

[3] Lee J, Singh J, Azamfar M. Industrial Artificial Intelligence. arXiv preprint arXiv:1908.02150. 2019 Aug 4.

[4] Vahid Dastjerdi A, Buyya R. Fog Computing: Helping the Internet of Things Realize. IEEE Comput Soc 2016:112-6.

[5] Lee J, Azamfar M, Singh J. A blockchain enabled Cyber-Physical System architecture for Industry 4.0 manufacturing systems. Manuf Lett 2019;20:34-9.

[6] Lee, J., Azamfar, M., Singh, J. and Siahpour, S., 2020. Integration of digital twin and deep learning in cyber-physical systems: towards smart manufacturing. IET Collaborative Intelligent Manufacturing, 2(1), pp.34-36.

[7] Taleb T, Samdanis K, Mada B, Flinck H, Dutta S, Sabella D. On Multi-Access Edge Computing: A Survey of the Emerging 5G Network Edge Cloud Architecture and Orchestration. IEEE Commun Surv Tutorials 2017;19:1657-81.

[8] Han S, I CL, Xu Z, Rowell C. Large-scale antenna systems with hybrid analog and digital beamforming for millimeter wave 5G. IEEE Commun Mag 2015;53:186-94.

[9] Bogale TE, Le LB. Massive MIMO and mmWave for 5G Wireless HetNet: Potential Benefits and Challenges. IEEE Veh Technol Mag 2016;11:64-75.

[10] Akyildiz IF, Wang P, Lin SC. SoftAir: A software defined networking architecture for 5G wireless systems. Comput Networks 2015;85:1-18.

[11] Liu J, Sheng M, Liu L, Li J. Network Densification in 5G: From the Short-Range Communications Perspective. IEEE Commun Mag 2017;55:96-102.

[12] Peng M, Li Y, Zhao Z, Wang C. System architecture and key technologies for 5G heterogeneous cloud radio access networks. IEEE Netw 2015;29:6-14.

[13] Abdelwahab S, Hamdaoui B, Guizani M, Znati T. Network function virtualization in 5G. IEEE Commun Mag 2016;54:84-91.

[14] Palattella MR, Dohler M, Grieco A, Rizzo G, Torsner J, Engel T, et al. Internet of Things in the 5G Era: Enablers, Architecture, and Business Models. IEEE J Sel Areas Commun 2016;34:510-27.

[15] Akyildiz IF, Nie S, Lin SC, Chandrasekaran M. 5G roadmap: 10 key enabling technologies. Comput Networks 2016;106:17-48.

[16] Bockelmann C, Pratas N, Nikopour H, Au K, Svensson T, Stefanovic C, et al. Massive machine-type communications in 5g: Physical and MAC-layer solutions. IEEE Commun Mag 2016;54:59-65. 
[17] Schulz P, Matthe M, Klessig H, Simsek M, Fettweis G, Ansari J, et al. Latency Critical IoT Applications in 5G: Perspective on the Design of Radio Interface and Network Architecture. IEEE Commun Mag 2017;55:70-8.

[18] Ashraf, Shehzad A., et al. "Ultra-reliable and low-latency communication for wireless factory automation: From LTE to 5G." 2016 IEEE 21st International Conference on Emerging Technologies and Factory Automation (ETFA). IEEE, 2016.

[19] Saberi S, Kouhizadeh M, Sarkis J, Shen L. Blockchain technology and its relationships to sustainable supply chain management. Int J Prod Res 2018;0:1-19.

[20] Khan MA, Salah K. IoT security: Review, blockchain solutions, and open challenges. Futur Gener Comput Syst 2018;82:395-411.

[21] Xu Q, Aung KMM, Zhu Y, Yong KL. A blockchain-based storage system for data analytics in the internet of things. Stud. Comput. Intell., vol. 715, Springer Verlag; 2018, p. 119-38.

[22] Samaniego, Mayra, and Ralph Deters. "Blockchain as a Service for IoT." 2016 IEEE International Conference on Internet of Things (iThings) and IEEE Green Computing and Communications (GreenCom) and IEEE Cyber, Physical and Social Computing (CPSCom) and IEEE Smart Data (SmartData). IEEE, 2016 .

[23] Shafagh H, Burkhalter L, Hithnawi A, Duquennoy S. Towards Blockchain-based Auditable Storage and Sharing of IoT Data. DlAcmOrg 2017.

[24] Azamfar, Moslem, Xiang Li, and Jay Lee. "Intelligent ball screw fault diagnosis using a deep domain adaptation methodology." Mechanism and Machine Theory 151 (2020): 103932.

[25] Dorri A, Steger M, Kanhere SS, Jurdak R. BlockChain: A Distributed Solution to Automotive Security and Privacy. IEEE Commun Mag 2017;55:119-25.

[26] Joshi NS, Singh S, Krugh M, Mears L. Background noise mitigation of dual microphone system for defect detection in electrical cable connection 2018;00.

[27] Vedant RM, Krugh M, Mears L. Measuring finger engagement during manual assembly operations in automotive assembly 2019;00.

[28] Azamfar M, Singh J, Bravo-Imaz I, Lee J. Multisensor data fusion for gearbox fault diagnosis using 2-D convolutional neural network and motor current signature analysis. Mechanical Systems and Signal Processing. 2020 Oct 1;144:106861.

[29] Peng M, Li Y, Jiang J, Li J, Wang C. Heterogeneous cloud radio access networks: A new perspective for enhancing spectral and energy efficiencies. IEEE Wirel Commun 2014;21:126-35. 